\title{
The confounding effect of systemic physiology on the hemodynamic response in newborns
}

Zimmermann, B B ; Roche-Labarbe, N ; Surova, A ; Boas, D A ; Wolf, M ; Grant, P E ; Franceschini, M A

\begin{abstract}
During neural activity, increases in glucose and oxygen consumption and release of vasoactive neurotransmitters cause a local increase in cerebral blood fl ow (CBF) [1] and cerebral blood volume (CBV). Increases in oxygen consumption are signifi-cantly lower than increases in $\mathrm{CBF}$ and as a result we see a net increase in the amount of oxygen in the blood and tissue [2]. Typical measures of neural activity in adults with near-infrared spectroscopy (NIRS) show a local increase in oxy-hemoglobin concentration (HbO) and a decrease in deoxyhemoglobin concentration (HbR), which corresponds to a local increase in BOLD signal measured with fMRI. In many neonatal functional studies inversions of these hemoglobin signals have been reported, across visual [3,4], olfactory [5], sensory-motor [6] and auditory [7] cortices. In general, the inversion starts at a few weeks of age. The reason for such an inversion in the functional hemodynamic signals is not yet understood. We hypothesize that changes in hematocrit during the transition from fetal to adult hemoglobin and the consequent period of low hematocrit cause such an inversion. To test this hypothesis, we performed a longitudinal auditory functional study in premature infants.
\end{abstract}

DOI: https://doi.org/10.1007/978-1-4614-1566-4_16

Posted at the Zurich Open Repository and Archive, University of Zurich

ZORA URL: https://doi.org/10.5167/uzh-70332

Book Section

Accepted Version

Originally published at:

Zimmermann, B B; Roche-Labarbe, N; Surova, A; Boas, D A; Wolf, M; Grant, P E; Franceschini, M A (2012). The confounding effect of systemic physiology on the hemodynamic response in newborns. In: Wolf, Martin; et al. Oxygen Transport to Tissue XXXIII. New York, NY: Springer, 103-109.

DOI: https://doi.org/10.1007/978-1-4614-1566-4_16 


\title{
The Confounding Effect of Systemic Physiology on the Hemodynamic Response in Newborns
}

\author{
B.B. Zimmermann, \\ Athinoula A. Martinos Center for Biomedical Imaging, Harvard Medical School, Massachusetts \\ General Hospital, 13th Street, Building 149, Charlestown, MA 02129, USA. Biomedical Optics \\ Research Laboratory, Clinic for Neonatology, University Hospital Zürich, Zürich, Switzerland

\section{N. Roche-Labarbe,} \\ Athinoula A. Martinos Center for Biomedical Imaging, Harvard Medical School, Massachusetts \\ General Hospital, 13th Street, Building 149, Charlestown, MA 02129, USA
}

\begin{abstract}
A. Surova,
Athinoula A. Martinos Center for Biomedical Imaging, Medical School, Massachusetts General Hospital, 13th Street, Building 149, Charlestown, MA 02129, USA

\section{D.A. Boas,}

Athinoula A. Martinos Center for Biomedical Imaging, Harvard Medical School, Massachusetts General Hospital, 13th Street, Building 149, Charlestown, MA 02129, USA

M. Wolf,

Biomedical Optics Research Laboratory, Clinic for Neonatology, University Hospital Zürich, Zürich, Switzerland
\end{abstract}

P.E. Grant, and Newborn Medicine and Radiology, Children's Hospital of Boston, Boston, MA, USA

\section{M.A. Franceschini}

Athinoula A. Martinos Center for Biomedical Imaging, Harvard Medical School, Massachusetts General Hospital, 13th Street, Building 149, Charlestown, MA 02129, USA

B.B. Zimmermann: bzim@mgh.harvard.edu

\section{Introduction}

During neural activity, increases in glucose and oxygen consumption and release of vasoactive neurotransmitters cause a local increase in cerebral blood fl ow (CBF) [1] and cerebral blood volume (CBV). Increases in oxygen consumption are signifi-cantly lower than increases in $\mathrm{CBF}$ and as a result we see a net increase in the amount of oxygen in the blood and tissue [2]. Typical measures of neural activity in adults with near-infrared spectroscopy (NIRS) show a local increase in oxy-hemoglobin concentration (HbO) and a decrease in deoxy-hemoglobin concentration $(\mathrm{HbR})$, which corresponds to a local increase in BOLD signal measured with fMRI. In many neonatal functional studies inversions of these hemoglobin signals have been reported, across visual [3, 4], olfactory [5], sensorymotor [6] and auditory [7] cortices. In general, the inversion starts at a few weeks of age.

The reason for such an inversion in the functional hemodynamic signals is not yet understood. We hypothesize that changes in hematocrit during the transition from fetal to

(C) Springer Science+Business Media, LLC 2012

Correspondence to: B.B. Zimmermann, bzimemgh . harvard. edu. 
adult hemoglobin and the consequent period of low hematocrit cause such an inversion. To test this hypothesis, we performed a longitudinal auditory functional study in premature infants.

\section{Materials and Methods}

We recruited six premature neonates from either the neonatal intensive care unit (NICU) or the Well Baby Nursery at the Massachusetts General Hospital (MGH) [26-33.4 weeks, mean 30 weeks gestational age (GA)]. All of the neonates had APGAR scores between 6 and 9 after $5 \mathrm{~min}$. We conducted a total of 18 recording sessions, with an average of three sessions per infant (range: $2-5$ ). The age of the infants at the time of each session ranged from 1 to 11 weeks of age, with a mean of 5.5 weeks [30-38 weeks, mean 35 weeks corrected gestational age (CGA)]. Our Institutional Review Board approved the study and parents provided informed consent.

All measurements were done at the infant's bedside. The protocol consisted of two parts: a measure of evoked hemodynamic changes in response to auditory stimuli (functional measurements) with a continuous-wave (CW-NIRS) system, and a measure of baseline hemoglobin concentration and oxygenation during rest (baseline measurements) with a frequency-domain system (FD-NIRS).

\subsection{Functional Measurements}

Auditory stimuli consisting of computer-generated repetitive syllables (e.g., "ma, ma, ma," or "bi, bi, bi") read by a female computer voice (AT\&T Labs, Inc., Florham Park, NJ) were presented in periods of $6 \mathrm{~s}$ followed by 6- to 16-s periods of silence (event-related presentation). Depending on cooperativeness of the baby, a session consisted of four runs of $250 \mathrm{~s}$ and 15 stimulation periods each. A sound-level meter allowed us to adjust the volume of the sound to a level of $60-65 \mathrm{~dB}$.

For the functional measurements, we used a commercial continuous-wave NIRS imaging system (CW4, TechEn Inc.) with 18 laser sources and 16 avalanche photo diode (APD) detectors [8]. Light from the sources is conducted to the functional probe and from there to the detectors by means of fiber-optic bundles. Each source fiber combines the light of a 690and an 830-nm laser. For these measurements, we used three source and six detector positions. The custom-made probe was made of black latex-free rubber and cushioning foam, and sources and detectors were arranged in two rows with a minimum source-detector distance of approximately $2.5 \mathrm{~cm}$. The functional probe was positioned over the left auditory cortex and fixed in position with sterile, self-adhesive elastic gauze. Data were acquired continuously during the functional runs and down-sampled to $10 \mathrm{~Hz}$.

\subsection{Baseline Measurements}

The baseline hemoglobin measurements were performed a few minutes after the functional measurements, with a customized near-infrared frequency-domain oxime-ter (ISS Inc., Champaign, IL, USA), as in $[9,10]$. The system includes 16 laser sources emitting at eight wavelengths with two redundant pairs $(659,2 \times 685,755,778,798$ and $2 \times 825 \mathrm{~nm})$ and two photomultiplier tube (PMT) detectors. The sources are modulated at $110 \mathrm{MHz}$, and the detectors at $110.005 \mathrm{MHz}$, to achieve heterodyne detection. Separation of the sources is achieved by rapid (10 ms) multiplexing. Two groups of eight lasers ( $\sim 1 \mathrm{~mW}$ power each) are combined into two source fiber bundles, and each detector is coupled to a fiber bundle. The fiber bundles are arranged in a row on a black rubber probe with source-detector distances of $1,1.5,2$, and $2.5 \mathrm{~cm}$. Multiple distances are necessary to quantify tissue optical properties and to derive hemoglobin concentration and saturation with this system. The probe was 
hand-held above the left-temporal position for $8 \mathrm{~s}$ and the measure was repeated 3-5 times as in [9].

\subsection{Data Analysis}

CW-NIRS data were analyzed with a new version of Homer (Homer 2) which, instead of working with a graphical user interface [11], is fully script-based and allows for comfortable batch processing of all measurements with the same parameters for an unbiased analysis. First the data are converted from light intensity to changes in optical density ( $\Delta \mathrm{OD})$. Segments of data showing rapid changes in optical density larger than a preset threshold of $1.5 \%$ are discarded as motion artifacts. Remaining evident motion artifacts are manually removed. If the motion artifacts occur during a stimulus period, that stimulus is removed from the average. Channels with low SNR or with Fourier spectra that do not show an arterial pulsation peak are removed. The $10 \mathrm{~Hz} \Delta \mathrm{OD}$ data are then band-pass-filtered between 0.02 and $0.5 \mathrm{~Hz}$ to remove slow drift and arterial and respiratory oscillations. To remove $0.1 \mathrm{~Hz}$ Mayer oscillations and remaining noise common to all channels, we performed principal component analysis (PCA) and removed principal components that described up to $75 \%$ of temporal covariance. Finally, the optical densities are converted to oxy and deoxy-hemoglobin concentration ( $\mathrm{HbO}, \mathrm{HbR})$ changes using the modified BeerLambert law and all stimulation sequences in a subject are block-averaged. To quantify the amplitude of $\mathrm{HbO}$ and $\mathrm{HbR}$ changes, we calculated the difference between the mean concentration of the last second of stimulation and the last second before stimulation. For every subject, the channel with the largest $\mathrm{HbO}$ absolute change is selected and reported and further analyzed.

Frequency domain multi-distance and multi-wavelength data are used to quantify baseline hemoglobin concentration and tissue oxygenation. To achieve standardized analysis of the FD-NIRS data, an automated routine developed in [10] was used. This script includes data quality assessment and rejection based on previously established statistical criteria. In particular, measurements were discarded if $R^{2}<0.9$ for the fit of the raw optical data (amplitude and phase) to the light transportation model, if $p>0.05$ for the fit of the absorption coefficients with the hemoglobin spectra, and for a linear fit of the reduced scattering coefficient versus wavelength.

\section{Results}

\subsection{Baseline Measurements}

In agreement with previous results $[9,10]$, we found that, at $\sim 4-6$ weeks of age, total hemoglobin concentration $(\mathrm{HbT})$ and $\mathrm{HbO}$ have a minimum corresponding to the minimum of hematocrit due to conversion of fetal to adult hemoglobin (see Fig. 16.1a, c). In contrast, HbR concentrations during the first 6 weeks of life remain constant (see Fig. 16.1b) probably because of two counterbalancing factors: decrease of hemoglobin in the blood because of faster depletion of fetal hemoglobin than formation of adult hemoglobin and increase of oxygen extraction fraction with adult relative to fetal hemoglobin. After this initial period $\mathrm{HbR}$ increases as $\mathrm{HbO}$ and $\mathrm{HbT}$. Consequently, tissue oxygenation $\left(\mathrm{StO}_{2}\right)$ decreases during the first 6 weeks of life and then becomes constant (see Fig. 16.1d ). As in our previous studies, we did not observe any correlation between any hemoglobin parameter and corrected gestational age.

\subsection{Functional Measurements}

We observed the expected increase in $\Delta \mathrm{HbO}$ with stimulation in only 7 out of 18 cases. The remaining 11 measurements showed a decrease in $\Delta \mathrm{HbO}$ with stimulation. In all but one case $\Delta \mathrm{HbT}$ followed $\Delta \mathrm{HbO}$ and in all but four cases $\Delta \mathrm{HbR}$ was inversely proportional to $\Delta$ 
$\mathrm{HbO}$. The inverted hemoglobin responses are not correlated with age or corrected gestational age (see Fig. 16.2b, c), but all occur between ages 3 and 8 weeks, when hematocrit and hemoglobin are low. Though for most of these babies we did not have a direct measure of hematocrit on the day of the measurement, we do have the measure of baseline cerebral hemoglobin and we can correlate it with the functional hemoglobin changes. If we divide the $\Delta \mathrm{HbO}$ responses into two groups, positive and negative, we found a strong correlation between the sign of the functional changes and the baseline total hemoglobin concentration ( $p$-value $=0.002$ for $\mathrm{HbT}$ (two-sample, two-tailed, unequal variance $T$-test), $p$-value $=0.01$ for $\mathrm{HbO}$ and $\mathrm{HbR}$ ). The $R^{2}$ of the scatter plot of $\Delta \mathrm{HbO}$ versus baseline $\mathrm{HbT}$ is 0.5 . More importantly we found that, for baseline HbT lower than 38 $\mu \mathrm{M}$ all $\Delta \mathrm{HbO}$ were negative, while for baseline $\mathrm{HbT}$ larger than $38 \mu \mathrm{M}$, all but one $\Delta \mathrm{HbO}$ were positive (see Fig. 16.2a). Results were similar when considering baseline $\mathrm{HbO}$ or $\mathrm{HbR}$. Results for $\Delta \mathrm{HbT}$ were consistent with the results for $\Delta \mathrm{HbO}$, while results for $\Delta \mathrm{HbR}$ were less statistically significant ( $p$-value $=0.026$ between $\Delta \mathrm{HbR}$ and baseline $\mathrm{HbR}$, larger than 0.05 for the $\mathrm{HbO}$ and $\mathrm{HbT}$ ) probably due to the smaller changes in $\Delta \mathrm{HbR}$ with respect to $\Delta \mathrm{HbO}$, and to consequent larger relative noise.

\section{Conclusions}

Our results show that the inversion in the functional hemodynamic responses in infants correlates with the total hemoglobin concentration. We used auditory stimuli which is known to cause neuronal activity in premature babies 30 weeks GA and older. While neural activity increases with age because of an increase in synapto-genesis and increasing synaptic density, an inversion of the hemodynamic responses is difficult to explain solely based on neural activity differences in the period 3-8 weeks of age. Our results suggest that while neural activity and metabolic demand increase with age, the available hemoglobin supply during the low hematocrit period is not sufficient to overcome oxygen demand during functional activation. We are developing a mathematical model to describe these results.

\section{Acknowledgments}

The authors thank all the families for their participation and the nurses, physicians, and staff in the Neonatal ICU, Special Care Nursery, Pediatric Neurology, and maternity units at the Massachusetts General Hospital. This work is supported by NIH grant R01 HD42908.

\section{References}

1. Fox PT, Raichlie ME, Mitun MA, et al. Nonoxidative glucose consumption during focal physiologic neural activity. Science. 1988; 241(4864):462-464. [PubMed: 3260686]

2. Malonek D, Grinvald A. Interactions between electrical activity and cortical microcir-culation revealed by imaging spectroscopy: implications for functional brain mapping. Science. 1996; 272(5261):551-554. [PubMed: 8614805]

3. Meek JH, Firbank M, Elwell CE, et al. Regional hemodynamic responses to visual stimulation in awake infants. Pediatr Res. 1998; 43(6):840-843. [PubMed: 9621996]

4. Kusaka T, Okubo K, Nagano K, et al. Activation of the visual cortex in newborn infants under natural sleep using multichannel near-infrared spectroscopy. Adv Exp Med Biol. 2003; 510:255259. [PubMed: 12580437]

5. Bartocci M, Winberg J, Ruggiero C, et al. Activation of olfactory cortex in newborn infants after odor stimulation: a functional near-infrared spectroscopy study. Pediatr Res. 2000; 48(1):18-23. [PubMed: 10879795]

6. Hintz SR, Benaron DA, Siegel AM, et al. Bedside functional imaging of the premature infant brain during passive motor activation. J Perinat Med. 2001; 29(4):335-343. [PubMed: 11565203]

7. Zaramella P, Freato F, Amigoni A, et al. Brain auditory activation measured by near-infrared spectroscopy (NIRS) in neonates. Pediatr Res. 2001; 49(2):213-219. [PubMed: 11158516] 
8. Franceschini MA, Fantini S, Thompson JH, et al. Hemodynamic evoked response of the sensorimotor cortex measured noninvasively with near-infrared optical imaging. Psychophysiology. 2003; 40(4):548-560. [PubMed: 14570163]

9. Franceschini MA, Thaker S, Themelis G, et al. Assessment of infant brain development with frequency-domain near-infrared spectroscopy. Pediatr Res. 2007; 61(5 Pt 1):546-551. [PubMed: 17413855]

10. Roche-Labarbe N, Carp SA, Surova A, et al. Noninvasive optical measures of CBV, StO(2), CBF index, and $\mathrm{rCMRO}(2)$ in human premature neonates' brains in the first six weeks of life. Hum Brain Mapp. 2010; 31(3):341-352. [PubMed: 19650140]

11. Huppert TJ, Diamond SG, Franceschini MA, et al. HomER: a review of time-series analysis methods for near-infrared spectroscopy of the brain. Appl Opt. 2009; 48(10):D280-D298.

[PubMed: 19340120] 

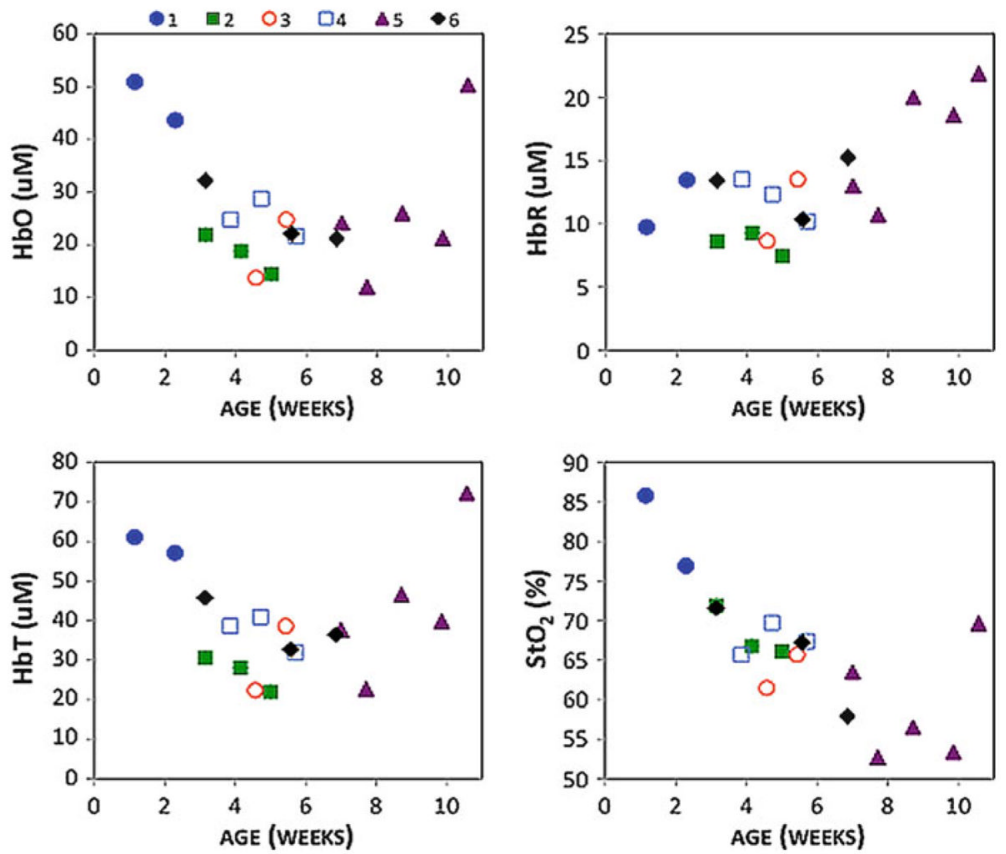

Fig. 16.1.

(a) Scatter plot of baseline oxy-hemoglobin ( $\mathrm{HbO}$ ) versus age, (b) deoxy-hemoglobin versus age, (c) total hemoglobin versus age, and (d) tissue oxygen saturation versus age. Different babies are indicated by different symbols 

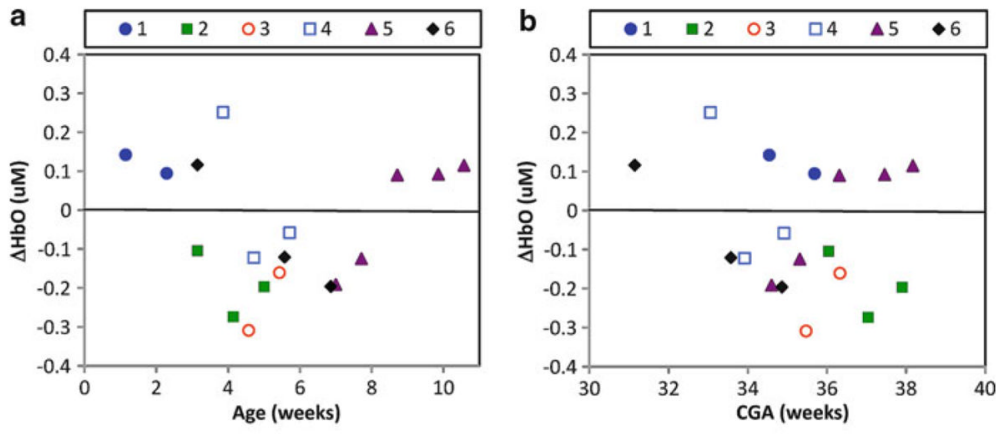

C

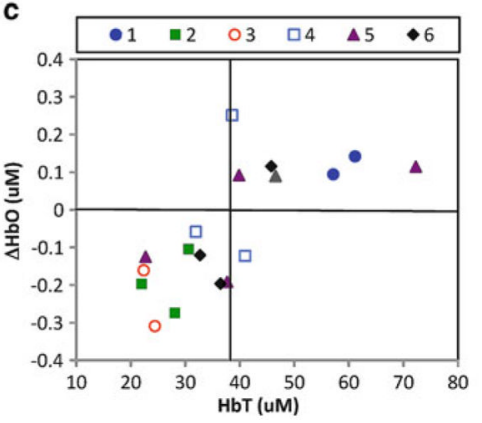

Fig. 16.2.

(a) Scatter plot of functional oxy-hemoglobin changes $(\Delta \mathrm{HbO})$ versus baseline total hemoglobin concentration (HbT), (b) $\Delta \mathrm{HbO}$ versus age, and (c) $\Delta \mathrm{HbO}$ versus corrected gestational age. Different babies are indicated by different symbols. A line through the zero $y$-axis is shown in all graphs. Also in (a) a dashed line at $38 \mu \mathrm{M} x$-axis divides the data in two groups: negative and positive $\Delta \mathrm{HbO}$ changes 